\title{
Identification of Socioeconomic Variables Responsible for Obesity Kidney Disease among Bangladeshi Adults
}

\section{K.C. Bhuyan}

Professor (Retired) of Statistics, Jahangir Nagar University, Dhaka, Bangladesh.

Corresponding Author: K.C. Bhuyan, Professor (Retired) of Statistics, Jahangir Nagar University, Dhaka, Bangladesh.

Received date: November 20, 2020; Accepted date: November 26, 2020; Published date: December 10, 2020

Citation: K.C. Bhuyan (2020) Identification of Socioeconomic Variables Responsible for Obesity Kidney Disease among Bangladeshi Adults $J$. Diabetes and Islet Biology 3(2); DOI:10.31579/2641-8975/0024

Copyright: (C) 2020 K.C. Bhuyan, This is an open access article distributed under the Creative Commons Attribution License, which permits unrestricted use, distribution, and reproduction in any medium, provided the original work is properly cited.

\begin{abstract}
The information presented here were the analytical results observed from data collected in investigating 995 Bangladeshi adults of 18 years and above. The objective of the investigation was to identify socioeconomic variables which enhance the health hazard obesity kidney disease. The analysis indicated that this health problem was noted among 6.2\% respondents and it was predominant among males, non-Muslims, elderly people, lower level educated adults, people belonged to families of medium economy, smokers, adults involved in sedentary activities, diabetic and hypertensive respondents. The prevalence rates among these respondents were $0.074,0.088,0.188,0.099,0.098,0.088$, $0.075,0.075$ and 0.186 , respectively. However, all these socioeconomic variables were not similarly responsible for obesity kidney disease among the adults. The most responsible variable was family expenditure followed by family income, physical labour, age, process food consumption, hypertension and duration of diabetes. These variables were identified by factor analysis.
\end{abstract}

Keywords: obesity; kidney disease; socioeconomic variables; association of socioeconomic variable with prevalence of obesity kidney disease; risk ratio; factor analysis

\section{Introduction}

Obesity, like hypertension and diabetes, is the major risk factor in developing kidney disease among human beings, especially among elderly people [1, 2, 3, 4, 5, and 6]. It increases the risk of chronic kidney disease (CKD) and end stage of renal disease (ESRD). It was noted that the elevated body mass index rapidly enhanced the CKD among the preexisting CKD patients and even among healthy men [2, 4]. The adverse impact of obesity on the kidney creates other complications, like nephrolithiasis and kidney malignancies. This is true for both men and women.

One of the cause of kidney failure is diabetes which is a condition characterized by high blood glucose levels and if this condition exists for long time it damages the millions of tiny filtering units within each kidney resulting kidney failure [7, 8]. It indicates that poor glucose control is a risk factor for kidney function alteration both in type-1 and type-2 diabetes [7]. Thus, it can also be said that the risk of kidney disease is associated with obesity, pre-diabetes and hypertension [8,9, 10, and 11]. The prevalence of obesity was increasing in many countries, especially in developing countries due to socio-demographic upward mobility despite continuing nutritional deficiencies $[12,13]$. In developed countries the problem of obesity has been increasing rapidly [12, 14, 15, and 16]. In 2016, the reported overweight adults of age 18 years and above were 1.9 billon and obese adults were 650 million [17].

The burden of obesity was shifting towards lower socioeconomic group of people most of whom are living in Asia-pacific region. Bangladesh is an Asian country and it has every chance of facing the problem of obesity and its consequences. Thus, it needs to study the proportion of obese people in Bangladesh and the proportion of obese kidney disease patients in the country. The specific objective of the present study is to identify the responsible socioeconomic variables for the prevalence of obesity and kidney disease simultaneously among the adults people of Bangladesh.

\section{Methodology}

The data for this research were collected by some nurses and medical assistants working in diagnostic centres. The centres were purposively selected from both urban and semi-urban areas of Bangladesh. The respondents of this study were 498 males and 497 females of age 18 years and above totalling 995 adults. This composition of male and female was considered to cover the national sex ratio 50.1: 49.9 of population of Bangladesh [18]. The investigated units were the residents of both urban and rural localities. As the adults were investigated from diagnostic centre, most $(67 \%)$ of them were found diabetic patients. The data were recorded during the session 2018-19.

The information of different socioeconomic variables of each selected respondent were recorded through a pre-designed and pre-tested questionnaire containing different questions related to residence, religion, gender, marital status, age, education, occupation, family income, family expenditure. Beside these demographic data, other information were on life-style, and prevalence of any of the noncommunicable diseases, duration of diabetes, and the stages of treatment of the disease including cost of treatment. Some of the socioeconomic variables were qualitative and some were quantitative in nature, but all the variables were noted in nominal scale for ease of analysis. The data of weight (in $\mathrm{kg}$ ) divided by height (in metre ${ }^{2}$ ) was used to measure the value of body mass index (BMI) to identify obese adults (if $\mathrm{BMI} \geq 27.5$; 
underweight, if $\mathrm{BMI}<18.5$; normal, if $18.5 \leq \mathrm{BMI}<23.0$; overweight, if $23.0<\mathrm{BMI}<27.5)[19,20]$. They were also divided into 4 groups according to their blood pressure (B.P) level ( $\mathrm{mmHg}$ ). The 4 groups were identified as optimal (if $\mathrm{BP}<120 / 80$ ), normal (if $\mathrm{BP}<130 / 85$ ), high normal (if BP < 140/90) and hypertensive (if BP $\geq 140 / 90$ ) [21, 22].

To fulfil the objective of the study, the association of each of the socioeconomic variable with simultaneous prevalence of obesity kidney disease was investigated, where significant association was decided if $\mathrm{p}$ value of any Chi-square statistic $\leq 0.05\left[\mathrm{P}\left(\chi^{2}\right) \leq 0.05\right]$. Irrespective of significant or insignificant association, the risk ratio and its confidence interval was calculated for adults for whom prevalence of obesity kidney problem was noted in higher rate for a particular level of a socioeconomic variable. Finally, factor analysis was done to select the most responsible factor for the prevalence of this health hazard. The most responsible factor was identified depending on the highest absolute value of factor loadings observed in doing factor analysis [23, 24, and 25]. All the calculations were done using SPSS Version 25.

\section{Results}

Total respondents were 995 , they were classified by level of obesity and prevalence of different health problem. Classified adults were shown in Table 1 below: Among the respondents $30.2 \%$ were obese, $12.4 \%$ had kidney problem and prevalence of obesity kidney disease was noted in $6.2 \%$ respondents. The percentage of obese adults suffering from kidney problem was 20.7. The percentages of adults of different health problems were in significantly increasing trend with the increase in level of body mass index $\left[\chi^{2}=275.875, \mathrm{p}\right.$-value $\left.=0.000\right]$.

\begin{tabular}{|c|c|c|c|c|c|c|c|c|c|c|}
\hline \multirow{3}{*}{$\begin{array}{c}\text { Health } \\
\text { problem }\end{array}$} & \multicolumn{8}{|c|}{ Level of obesity } & \multicolumn{2}{|c|}{ Total } \\
\hline & \multicolumn{2}{|c|}{ Under weight } & \multicolumn{2}{|c|}{ Normal } & \multicolumn{2}{|c|}{ Overweight } & \multicolumn{2}{|c|}{ Obese } & \multirow[t]{2}{*}{ Number } & \multirow[t]{2}{*}{$\%$} \\
\hline & Number & $\%$ & Number & $\%$ & Number & $\%$ & Number & $\%$ & & \\
\hline None & 33 & 5.4 & 181 & 29.9 & 324 & 53.5 & 68 & 11.2 & 606 & 60.9 \\
\hline Heart & 3 & 2.4 & 16 & 12.7 & 42 & 33.3 & 65 & 51.6 & 126 & 12.7 \\
\hline Eye & 1 & 0.8 & 22 & 17.9 & 25 & 20.3 & 75 & 61.0 & 123 & 12.4 \\
\hline Kidney & 1 & 1.1 & 10 & 10.9 & 19 & 20.7 & 62 & 67.4 & 92 & 9.2 \\
\hline Others & 0 & 0.0 & 4 & 8.3 & 14 & 29.2 & 30 & 62.5 & 48 & 4.8 \\
\hline Total & 38 & 3.8 & 233 & 23.4 & 424 & 42.6 & 300 & 30.2 & 995 & 100.0 \\
\hline
\end{tabular}

Table 1: Distribution of adults by level of obesity and prevalence of health problem

In the sample there were $53.4 \%$ urban residents and $6.6 \%$ of them were suffering from obesity kidney disease [Table 2]. Urban and rural adults were almost at similar risk of this health hazard [R.R. $=1.13$, C.I. $(0.69$, 1.84]. Residence had no significant impact on obesity kidney problem [ $\chi^{2}=0.263, \mathrm{p}-$ value $\left.=0.615\right]$. Gender variation was also not a significant factor for obesity kidney disease [ $\chi^{2}=3.446$, p-value=0.117]. The percentage of male respondents was 51.1 and $7.4 \%$ of them were the patients of obesity kidney problem. They were at $48 \%$ more risk of the disease [R.R. $=1.48$, C.I. $(0.90,2.67)]$. Non-Muslim adults were only $14.8 \%$, but higher percentage of them $(8.8 \%)$ were facing the problem of this health hazard. They were at $60 \%$ more risk of the problem [R.R $=1.60$, C.I. $(0.95,2.42)]$. However, obesity kidney disease was independent of religion $\left[\chi^{2}=2.015, \mathrm{p}-\right.$ value $\left.=0.156\right]$. Married $(93.1 \%)$ and single adults were at similar risk of this health hazard, though percentage $(6.3 \%)$ of affected married adults compared to affected single person $(5.8 \%)$ was slightly more [R.R.=1.08, C.I. $(0.51,2.87)]$. Marital status was not an influencing factor for obesity kidney disease $\left[\chi^{2}=0.194, \mathrm{p}-\right.$ value $=0.877$. Among the respondents $8 \%$ were of age 60 years and above and $18.8 \%$ of them were suffering from obesity kidney disease. Their risk for this health problem was 3.65 times as it was for others [R.R. =3.65, C.I. $(1.96,6.79)]$. Again, $19.6 \%$ were of age 50 years and above. The percentage of sufferers in these group was 16.9 and their risk for this health problem was 4.67 times as it was for others [R.R. $=4.67$, C.I. (2.91, 7.50)]. With the increase in age of adults there was a significant increasing trend in the percentage of affected adults $\left[\chi^{2}=66.281, \mathrm{p}\right.$-value $=0.000]$.

Though level of education had no statistically significant impact on obesity kidney disease, lower level education and no education together was more risk creating factor for the disease [R.R. $=1.64$, C.C. $(0.96,2.50)$; $\chi^{2}=0.056, \mathrm{p}-$ value $\left.=0.056\right]$. There were $17.8 \%$ adults having no education and primary education and $9.0 \%$ of these two groups were facing the problem of this health hazard. Higher educated people $(57.5 \%)$ were also more affected $(6.6 \%)$ by this disease compared to the affected adults observed in the sample. The adults of different professions were almost similarly affected by the disease $\left[\chi^{2}=0.261, \mathrm{p}-\right.$ value $\left.=0.992\right]$. However, the percentage of affected service persons and retired persons together $(42.9 \%)$ were slightly more $(6.6 \%)$ compared to the overall percentage of affected adults. Even these two groups of adults had similar risk of the problem [R.R. $=1.10$, C.I. $(0.68,1.79)]$. The percentage of adults who did not do any physical labour was 51.7 and $6.4 \%$ of them were the patients of this health problem. For them the risk of the disease was almost similar as it was for others [R.R. $=1.18$, C.I. $(0.72,1.93)]$. But physical activity was not associated with the prevalence of obesity kidney disease [ $\chi^{2}=0.65$, p-value $\left.=0.799\right]$.

Income $\left[\chi^{2}=1.890, \mathrm{p}\right.$-value $\left.=0.595\right]$ and expenditure $\left[\chi^{2}=4.06, \mathrm{p}-\right.$ value $=0.203]$ had no influence on obesity kidney disease and there was no trend in the rates of affected persons with the increase in level on income or expenditure. But, higher percentage $(9.8 \%)$ of adults of families having income taka 100 - 150 thousand $(6.1 \%)$ were affected by this health problem. For them the risk of the disease was $64 \%$ more as it was for others $[R . R .=1.64$, C.I. $(0.74,3.66)]$. Similar higher risk was observed for adults of families spending taka $60-80$ thousand per month as family expenditure [R.R. $=1.74$, C.I. $(0.99,2.97)]$. The respondents of this group were $14.4 \%$ and affected adults of this group were $9.8 \%$. The percentage of smokers was 33.1 and $7.5 \%$ of them were patients of obesity kidney disease. Smoking habit was significantly associated with the prevalence of obesity kidney problem and smokers had $89 \%$ more risk of this health hazard compared to the risk of non-smokers $\left[\chi^{2}=5.615, \mathrm{p}-\right.$ value $=0.018 ;$ R.R. $=1.89$, C.I. $(1.16,3.07)]$. 


\begin{tabular}{|c|c|c|c|c|c|c|}
\hline \multirow{3}{*}{ Socioeconomic variables } & \multicolumn{4}{|c|}{ Prevalence of obesity kidney disease } & \multirow{2}{*}{\multicolumn{2}{|c|}{ Total }} \\
\hline & \multicolumn{2}{|c|}{ Yes } & \multicolumn{2}{|c|}{ No } & & \\
\hline & Number & $\%$ & Number & $\%$ & Number & $\%$ \\
\hline \multicolumn{7}{|l|}{ Residence } \\
\hline Rural & 35 & 6.6 & 496 & 93.4 & 531 & 53.4 \\
\hline Urban & 27 & 5.8 & 437 & 94.2 & 464 & 46.6 \\
\hline Total & 62 & 6.2 & 933 & 93.8 & 995 & 100.0 \\
\hline \multicolumn{7}{|l|}{ Gender } \\
\hline Male & 37 & 7.4 & 461 & 92.6 & 498 & 50.1 \\
\hline Female & 25 & 5.0 & 472 & 95.0 & 497 & 49.9 \\
\hline \multicolumn{7}{|l|}{ Religion } \\
\hline Muslim & 49 & 5.8 & 799 & 94.2 & 848 & 85.2 \\
\hline Non-Muslim & 13 & 8.8 & 134 & 91.2 & 147 & 14.8 \\
\hline \multicolumn{7}{|l|}{ Marital status } \\
\hline Currently married & 58 & 6.3 & 868 & 93.7 & 926 & 93.1 \\
\hline Currently single & 4 & 5.8 & 65 & 94.2 & 69 & 6.9 \\
\hline \multicolumn{7}{|l|}{ Age (in years) } \\
\hline$<25$ & 3 & 1.5 & 193 & 98.5 & 196 & 19.7 \\
\hline $25-40$ & 6 & 1.5 & 395 & 98.5 & 401 & 40.3 \\
\hline $40-50$ & 20 & 9.9 & 183 & 90.1 & 203 & 20.4 \\
\hline $50-60$ & 18 & 15.7 & 97 & 84.3 & 115 & 11.6 \\
\hline $60^{+}$ & 15 & 18.8 & 65 & 81.2 & 80 & 8.0 \\
\hline \multicolumn{7}{|l|}{ Education } \\
\hline Illiterate & 5 & 7.7 & 60 & 92.3 & 65 & 6.5 \\
\hline Primary & 12 & 9.9 & 109 & 90.1 & 121 & 12.2 \\
\hline Secondary & 7 & 3.0 & 0 & 97.0 & 237 & 23.8 \\
\hline Higher & 38 & 6.6 & 534 & 93.4 & 572 & 57.5 \\
\hline \multicolumn{7}{|l|}{ Occupation } \\
\hline Agriculture and unemployed & 6 & 5.8 & 98 & 94.2 & 104 & 10.5 \\
\hline Business & 15 & 6.4 & 219 & 93.6 & 234 & 23.5 \\
\hline Service and skilled labor & 20 & 6.6 & 285 & 93.4 & 305 & 30.7 \\
\hline Retire & 8 & 6.6 & 114 & 93.4 & 122 & 12.3 \\
\hline Housewife & 13 & 5.7 & 217 & 94.3 & 230 & 23.1 \\
\hline \multicolumn{7}{|l|}{ Income (000 taka ) } \\
\hline$<50$ & 23 & 5.9 & 366 & 94.1 & 389 & 39.1 \\
\hline $50-100$ & 27 & 6.4 & 393 & 93.6 & 420 & 42.2 \\
\hline $100-150$ & 14 & 9.8 & 47 & 90.2 & 61 & 6.1 \\
\hline $150^{+}$ & 6 & 4.8 & 119 & 95.2 & 125 & 12.6 \\
\hline \multicolumn{7}{|l|}{ Family expenditure (in 000 taka) } \\
\hline$<40$ & 25 & 6.0 & 391 & 94.0 & 416 & 41.4 \\
\hline $40-60$ & 18 & 6.0 & 284 & 94.0 & 302 & 30.4 \\
\hline $60-80$ & 14 & 9.8 & 129 & 90.2 & 143 & 14.4 \\
\hline $80^{+}$ & 5 & 3.7 & 129 & 96.3 & 134 & 13.5 \\
\hline \multicolumn{7}{|l|}{ Smoking habit } \\
\hline Yes & 29 & 8.8 & 300 & 91.2 & 329 & 33.1 \\
\hline No & 33 & 5.0 & 633 & 95.0 & 666 & 66.9 \\
\hline \multicolumn{7}{|l|}{ Habit of taking process food } \\
\hline Yes & 25 & 6.9 & 338 & 93.1 & 363 & 36.5 \\
\hline No & 37 & 5.9 & 597 & 94.1 & 632 & 63.5 \\
\hline Physical work & & & & & & \\
\hline Yes & 29 & 6.0 & 452 & 94.0 & 481 & 48.3 \\
\hline No & 33 & 6.4 & 481 & 93.6 & 514 & 51.7 \\
\hline Involved in sedentary activity & & & & & & \\
\hline Yes & 33 & 7.5 & 409 & 92.5 & 442 & 44.4 \\
\hline No & 29 & 5.2 & 534 & 94.8 & 553 & 55.6 \\
\hline Level of blood pressure & & & & & & \\
\hline Optimum & 11 & 2.0 & 529 & 98.0 & 540 & 54.3 \\
\hline Normal & 22 & 7.8 & 258 & 92.2 & 280 & 28.1 \\
\hline High normal & 18 & 15.5 & 98 & 84.5 & 116 & 11.7 \\
\hline Hypertensive & 11 & 18.6 & 48 & 81.4 & 59 & 5.9 \\
\hline
\end{tabular}




\begin{tabular}{|c|c|c|c|c|c|c|}
\hline \multicolumn{7}{|c|}{ Prevalence of diabetes } \\
\hline Yes & 50 & 7.5 & 617 & 92.5 & 667 & 67.0 \\
\hline No & 12 & 3.7 & 316 & 96.3 & 328 & 33.0 \\
\hline \multicolumn{7}{|c|}{ Duration of diabetes ( in years) } \\
\hline Does not arise & 12 & 3.7 & 316 & 96.3 & 328 & 33.0 \\
\hline$<5$ & 5 & 1.7 & 286 & 98.3 & 291 & 29.2 \\
\hline $5-10$ & 13 & 6.3 & 193 & 93.7 & 206 & 20.7 \\
\hline $10-15$ & 13 & 13.1 & 86 & 86.9 & 99 & 9.9 \\
\hline $15^{+}$ & 8 & 18.2 & 63 & 81.8 & 71 & 7.1 \\
\hline Total & 62 & 6.2 & 933 & 93.8 & 995 & 100.0 \\
\hline
\end{tabular}

Table 2: Distribution of adults according to prevalence of obesity kidney disease and socioeconomic variables

The percentage of process food consumers was 36.5. The prevalence of obesity kidney disease was noted among $6.9 \%$ of them. The risk of prevalence in them was only $18 \%$ more as it was in non-consumers of process food [R.R. $=1.18$, C.I. $(0.72,1.93)]$. But habit of process food consumption was not significantly associated with prevalence of obesity kidney disease $\left[\chi^{2}=0.65, \mathrm{p}-\right.$ value $\left.=0.799\right]$. The percentage of adults involved in sedentary activity was 44.4 and $7.5 \%$ of them were the patients of obesity kidney disease. The chance of prevalence in them was $54 \%$ more as it was in others [R.R. $=1.54$, C.I. $(0.95,2.49)]$. However, prevalence of the disease was statistically independent of involvement in sedentary activity [ $\chi^{2}=2.076, \mathrm{p}$-value $\left.=0.150\right]$.

The sample hypertensive adults were $5.9 \%$ and prevalence of obesity kidney disease was observed among $18.6 \%$ of them. The risk of prevalence in them was 3.42 times as it was in others R.R. $=3.42$, C.I. $(1.88,6.46)]$. The rate of prevalence of this health hazard was significantly increasing with the increase in level of blood pressure [ $\chi^{2}$ $=50.203, \mathrm{p}-$ value $=0.000]$. Among the respondents $67 \%$ were diabetic patients. The prevalence of obesity kidney disease was noted in $7.5 \%$ of them. The chance of prevalence in them was 2.05 times as it was in others [R.R. = 3.42, C.I. $(1.11,3.790]$. Prevalence of diabetes and prevalence this health hazard was significantly associated $\left[\chi^{2}=4.857, \mathrm{p}\right.$-value= $0.018]$. With the increase in duration of diabetes there was significant increase in the rate of prevalence of obesity kidney disease $\left[\chi^{2}=87.715\right.$, $\mathrm{p}$-value $=0.000]$. The rate was highest $(18.2 \%)$ among diabetic patients (4.4) suffering for 15 years and more. The chance of prevalence in them was 3.20 times as it was in others [R.R. $=3.20$, C.I. $(1.62,5.81)]$.

\section{Factor Analysis}

The study of association of prevalence of obesity kidney disease with socioeconomic variable showed that age, smoking habit, sedentary activity, prevalence of diabetes, duration of diabetes and blood pressure level were significantly influencing factors. But none of these variables could be identified as most responsible one for the prevalence. To identify the most responsible variable factor analysis was done utilizing all the studied variables. But final factor analysis was done using the variables which showed at least 0.50 as communality during analysis. The final included variables were age, family income, family expenditure, habit of consumption of process food, physical work, duration of diabetes and blood pressure level. These included variables were satisfactory as KMO $=0.705$ which gave $\chi^{2}=22.710$ with $\mathrm{p}$-value $=0.000$. The variables explained $72.353 \%$ variation in the data set. The analysis showed that family expenditure was the most responsible variable for the prevalence of obesity kidney disease followed by family income, physical work, and age, consumption of process food, blood pressure level and duration of diabetes. The results of factor analysis were shown in Table 3.

\begin{tabular}{|l|l|l|l|l|}
\hline \multirow{2}{*}{ Variables } & \multicolumn{2}{|c|}{ Communality } & \multicolumn{2}{c|}{ Coefficient of } \\
\cline { 2 - 5 } & Initial & Final & Factor - 1 & Factor -2 \\
\hline Residence & 0.060 & - & - & - \\
\hline Religion & 0.004 & - & - & - \\
\hline Gender & 0.809 & - & - & - \\
\hline Age & 0.658 & 0.796 & 0.546 & 0.706 \\
\hline Marital status & 0.064 & - & - & - \\
\hline Education & 0.486 & - & - & - \\
\hline Occupation & 0.670 & - & - & - \\
\hline Family income & 0.809 & 0.822 & -0.827 & 0.387 \\
\hline Family expenditure & 0.822 & 0.875 & -0.876 & 0.330 \\
\hline Smoking habit & 0.685 & - & - & - \\
\hline Sedentary activity & 0.444 & - & - & - \\
\hline Physical work & 0.636 & 0.676 & 0.808 & -0.149 \\
\hline Consumption of process food & 0.539 & 0.551 & -0.472 & 0.573 \\
\hline Blood pressure & 0.516 & 0.718 & 0.465 & 0.709 \\
\hline Prevalence of diabetes & 0.401 & - & - & - \\
\hline Duration of diabetes & 0.738 & 0.615 & 0.436 & 0.651 \\
\hline
\end{tabular}




\section{Discussion}

Overweight and obesity are associated with chronic kidney disease [26, 27, and 28]. This phenomenon is observed even among non-diabetic patients [29]. Besides overweight and obesity, other related variables are lifestyle factors such as smoking habit, physical activity, fast food consumption, diet quality, and diabetes, hypertension [7, 30, and 31]. The other socioeconomic variables are also expected to be associated with CKD as these are associated with obesity [32]. The socioeconomic variables may have impacts on simultaneous prevalence of obesity kidney disease. This hypothesis lead to identify some of the responsible socioeconomic variables for prevalence of this health hazard. For this, investigation was conducted on 995 adults of 18 years and above residing in both urban and rural areas of Bangladesh.

From the analysis of the data it was observed that obesity kidney disease was prevailed among $6.2 \%$ adults. Some of the socioeconomic variables were significantly associated with prevalence of the disease. These are age, smoking habit, prevalence of diabetes, duration of diabetes and blood pressure. The prevalence rate was $16.9 \%$ among elderly people of age 50 years and above (19.6\%). The risk of the disease was more than 4 times for this group of adults. The prevalence rate was $8.8 \%$ among smokers $(33.1 \%)$. The chance of prevalence was $89 \%$ more compared to that of non-smokers. Among diabetic adults $(67 \%)$ the prevalence rate was $7.5 \%$ and they were two times exposed to this health hazard compared to nondiabetic adults. The percentage of adults suffering from diabetes for 15 years and above was 4.4. For them the risk of prevalence was more than 3 times as it was for others. Percentage of hypertensive adults was 5.9 and prevalence rate was $18.6 \%$ in them. Their risk of prevalence was 3.42 times as it was in others.

Religion was independent of prevalence of obesity kidney disease but non-Muslims (14.8\%) were $60 \%$ more exposed to this health hazard. Family income and family expenditure were not significantly associated with this health problem, but adults $(6.1 \%)$ belonged to medium income (Taka $100-150$ thousand per month) had $64 \%$ more risk of prevalence. The chance of prevalence of the disease was $74 \%$ more for adults $(14.4 \%)$ belonged to families spending $60-80$ thousand taka. The percentage of adults involved in sedentary activity was 44.4 and they were more exposed to this health problem by $54 \%$. The risk of prevalence of obesity kidney disease was almost similar for adults irrespective of gender, residence, marital stats, physical work, and process food consumption.

Some variables were found significantly associated with prevalence of obesity kidney disease and some were responsible, though not significantly associated with prevalence, for enhancing the risk of prevalence. However, the most responsible variable for the prevalence was family expenditure followed by family income, physical activity, age, process food consumption, blood pressure, and duration of diabetes. This was noted from factor analysis.

\section{Conclusion}

The present analysis was an attempt to identify some socioeconomic variables responsible for prevalence of obesity kidney disease among Bangladeshi adults of age 18 years and above. The adults visiting some diagnostic centres located in urban and semi-urban areas in Bangladesh were the investigating units and their number was 995 . The data on residence, religion, gender, marital status, age ,education, occupation, family income, family expenditure, physical activity, food habit, smoking habit, prevalence of diabetes, duration of diabetes, blood pressure level including the different steps of treatment of needed for recovery of the health problem were recorded.

The sample contained $51.1 \%$ males, $53.4 \%$ urban people, $14.8 \%$ nonMuslims, $19.6 \%$ elderly people, $18.7 \%$ lower educated adults, $33.1 \%$ smokers, $51.7 \%$ physically inactive adults, $36.5 \%$ process food consumers, $67.0 \%$ diabetic patients, $5.9 \%$ hypertensive adults and $44.4 \%$ adults who were involved in sedentary activity. The prevalence of obesity kidney disease was noted among 6.2\% adults. This rate was more than $6.2 \%$ among all the above classified adults. However, the chance of prevalence was $48 \%$ more among males, $60 \%$ more among non-Muslims, $367 \%$ more among elderly people, $64 \%$ more among lower educated people, $89 \%$ more among smokers, $54 \%$ more among adults involved in sedentary activity, $105 \%$ more among diabetic patients, and $242 \%$ more among hypertensive adults. The chance was $220 \%$ more among adults suffering from diabetes for 15 years and above. These results were noted in calculating risk ratio. However, the identified most responsible factor for the prevalence was medium family expenditure, followed by medium family income, physical inactivity, over age, consumption of process food, hypertension, and longer duration of diabetes. This conclusion was drawn on the basis of risk ratio and higher absolute value of factor loading.

The prevalence of overweight and obesity are in increasing trend over the last decades in both developed and developing countries [6, 32, 33, 34, 35, and 36]. Prevalence has implications in increasing the rate of noncommunicable diseases including kidney diseases [1, 2, 3, 6, and 32]. Hence, strategies to decrease CKD need the inclusion of prevention technique of overweight and obesity [37]. But due to upward social mobility among the people of Bangladesh, the influences of obesity cannot be avoided but the intensity of the problem can be reduced to a great extent if proper action plan is formulated by the health planners to implement their plan in both urban and rural localities [38]. The social workers, medical practitioners, and other health workers can do a lot to encourage the people in leading healthy life. For this, people can be advised to avoid

(i) Process food, fatty and salty food, can food,

(ii) Sedentary activity,

(ii) Gain of unwanted body weight.

People can also be advised to do some sort of physical work and physical exercise if and when these are possible.

\section{References}

1. Hall, M.E.; do Carmo, J.M.; and Hall, J.E. (2014): Obesity, hypertension, and chronic kidney disease, Inter. J. of Nephro and Renovas Dis, 7, 75 - 88.

2. Foster, M.C.; Hwang.S. J; Larson, M.G.; Lichtman, j.h.; Nisha, I.P.; et al. (2008): Overweight, obesity and the development of stage 3 chronic kidney disease: the Framingham Heart Study: Amer J. Kidney Disease, 52(1), 39 - 48.

3. Lu, J.L.; Molnar, M.Z.; Naseer, A.; Mikkelsen, M.K.; KalantarZadeh, K. and Kovesley, C.P.(2015): Association of age and BMI with kidney function and mortality : a cohort study, Lancet Diabetes Endocrinolgy,3, 704 - 714.

4. Tsujimoto, T.; Sairenchi, T.; Iso, H.; Irie, F.; Yamagishi,K.; et al. (2014): The dose response relationship between body mass index and the risk of stage $\geq 3$ chronic kidney disease in a general Japanese population, The Ibraki prefectural health study, $J$. Epidemiology 24(6), 444-451.

5. Lu, J.L.; Kalantar- Zadeh,K.; Ma, J.Z.; Quarles, L.D. and Kovesdy, C.P. (2014): Association of body mass index with outcomes in patients with CKD , J. Amer Soc Nephrol, 25, 2088 $-2096$.

6. Casaba, P.K.; Furth, S.L. and Zaccali, C. (2017): Obesity and kidney disease: Hidden consequences of the epidemic, Amer J. Hypertension, 30(3), 328 - 336. 
7. Bhuyan, K.C.(2020): Identification of socioeconomic variables responsible for diabetic kidney disease among Bangladeshi adults, Biomed J Sci \& Tech Rese., 24(2), 18092 - 18098.

8. Echouffo - Tcheugui, J.B.; Narayan, K.M.; Weisman, D.; Golden, S.H. and Jaar, B.G. (2016): Association between prediabetes and risk of chronic kidney disease: a systematic review and meta-analysis, Diabetic Medicine, 33(12), 1615

9. Deloitte (2011): Two of a Kind, Kidney Health Australia.

10. Hall, J.H.; Brands, D.W.; Henegar, J.R; et al (1998): Kidney function as a cause and a consequence of obesity hypertension, Clin Exp Pharmacol Physiol, 25, 58 - 64.

11. Kriska, A.M.; Laporte, R.E.; Patnick, S.L. et al (1991): The association of physical activity and diabetic complications I individuals with insulin dependent mellitus: the epidemiology of diabetes complications study vii. J. Clin Epidemiology, 44.

12. Bertino, M.; Ardiri, A.M.; Ali, F.T.; Boemi, P.M.S.; Cilio, D. et al (2006): Obesity and related disease: an epidemiological study in Eastern Sicily, Minerva Gastroenterol Dietol, 52(4), 379 385 .

13. Calamusa, G.; Amodio, E; Costantino, C.; Maria,D.; Pasquate, et al (2012): Body mass index and factors associated with overweight and obesity : a cross-sectional study of adult subjects living in a small city of Western Sicily (Italy), Italian J. of Pub. Health, 9(3), e539.

14. Bhuyan, K.C. and Jannatul, F, (2019): Discriminating Bangladeshi adults by level of obesity, LOJ Med Sci, 3(1), $184-$ 191.

15. Hu, F.B. et al (2000): Body mass index and cardiovascular risk factors in a rural Chinese Population, Amer J. Epideo, 151, 88 97.

16. Stamler, J. (1991): Epidemiological findings on body mass and blood pressure in adults, Annals of Epidemiology, 1, 347 - 362.

17. Bhuyan, K.C. (2020): Discriminating Bangladeshi adults by the prevalence of diabetic hypertension, J. Dia Metab, 11.851.

18. Bangladesh Bureau of Statistics (2018): Statistical Year Book of Bangladesh, 2017, BBS, Dhaka, Bangladesh.

19. Appropriate Body Mass Index for Asian Population and its Implications for Policy and Intervention Strategies, WHO Expert Consultation, Public Health, Lancet 363, 2004.

20. Biswas, T.; Garnett, P. Sarah and Rawal, B.Lal (2017): The prevalence of underweight, overweight, and obesity in Bangladesh: Data from a national survey, PLoS One, 12(5), e0177395.

21. Jan, A.S.; Yan, Li.; Azusa, H.; KEI, A.; Eamon, D. and O'Brien, E.: (2017) Blood pressure measurement anno 2016. Amer J. Hypertens, 30(5), 453 - 463.

22. Jessica, Y.I.;Zaman, M.M.; Haq, S.A.; Ahmed, S. and Al-Quadir ,Z. ( 2018): Epidemiology of hypertension among Bangladeshi adults using the 2017 ACC/AHA Hypertension Clinical Guidelines and Joint National Committee 7 Guideline, $J$. Hypertens, 32, $668-680$.
23. Bhuyan, K.C. (2019): A note on factor analysis applied in medical research, Archives in Biomed Eng. And Biotech. 1(4), 1-3.

24. Yotoka, T. (1983): Some criteria for variable selection in factor analysis, Behaviormetrika, 13, 31 -45.

25. Rusico, J. and Roche, B. (2012): Determining the number of factors to retain in an exploratory factor analysis using comparison data of known factorial structure, Psychological Assessment, 24(2), $282-292$.

26. Kramer, H.; Luke, A.; Bidani, A.; Cao, G.; Cooper, R. and McGee, D. (2005): Obesity and prevalent and incident CKD: the hypertension detection and follow-up program, Amer J. Kidney Disease, 46(4), 587 - 594.

27. Zoccali, C. (2009): Overweight, obesity and metabolic alterations in chronic kidney disease, Prilozi, 30(2), $17-31$.

28. Garofalo, C.; Borrelli, S.; Minutolo, R.; Chiodini, P.; De Nicola, L. and Conte, G. (2017): A systematic review and meta-analysis suggests obesity predicts onset of chronic kidney disease in the general population, Kidney Int. 91(5), $1224-1235$.

29. Othman. M.; Kawar, B. and El Nahas, A.M. (2009): Influence of obesity on progression of non-diabetic chronic kidney disease: a retrospective cohort study, Nephrol Clin Pract., 113(1), 16 - 23.

30. Chang, A.; Horn, L.V.; Jacobs Jr., D.R.; Liu, K.; et al. (2013): Lifestyle-related factors, obesity, and incident microalbuminuria: the CARDIA Study, Amer J. Kidney Disease, 62(2), $267-275$.

31. Bhuyan, K.C. and Fardus, J (2019): Level of obesity and socioeconomic factors of a group of adult people of Bangladesh: A factor analysis approach, Amer Jour Data Min and Knowle Discovery, 4(1), 8 - 14.

32. Bhuiyan, D. and Bhuyan, K.C. (2019): Discriminating Bangladeshi adults by non-communicable diseases, Rehabilitation Sci., 4(3), 35 - 43.

33. WHO (2020): Fact Sheets/ Detail/ Obesity and Overweight, March 2020.

34. Dhurandhar, N.V.; (2004) Contribution of pathogene in human obesity. Drug News \& Perspectives; 7(5), 307-313.

35. Berg, C; Rosengren, A; Aires, N; et al; (2005) Trends in overweight and obesity from 1985 to 2002 in Goteborg, west Sweden. International Journal of Obesity, 29 (8), 916 - 924.

36. Skliros, E.A.; Merkoures.P; Sotiropoulos, A, et al. (2008) The relationship between body mass index and hypertension in elderly Greeks; the Nemea Primary Care Study. J. of the American Geriatrics Society, 56(5), 954-955.

37. Gelber, R.P.; Kurth, T.; Kausz, A.T.; Manson, J.E.; Burning, J.E.; Levy, A.S. and Gaziano, J.M. (2005): Association between BMI and CKD in apparently healthy men, Amer Jour Kidney Disease, 46, $871-880$.

38. Bhuyan, K.C. (2020): Identification of risk factors for obesity retinopathy among Bangladeshi adults, Current Research in Diabetes \& Obesity jour., 13(5), $0064-0071$. 